\title{
Geomorphic identification of physical habitat features in a large, altered river system
}

\author{
Lucie Guertault ${ }^{1, *}$, Garey Fox ${ }^{1}$, and Shannon Brewer ${ }^{2}$ \\ ${ }^{1}$ Department of Biological and Agricultural Engineering, North Carolina State University, Raleigh, \\ North Carolina, USA \\ ${ }^{2}$ U.S. Geological Survey, Oklahoma Cooperative Fish and Wildlife Research Unit, Oklahoma State \\ University, Stillwater, Oklahoma, USA
}

\begin{abstract}
Altered flow regimes in streams can significantly affect ecosystems and disturb ecological processes, leading to species loss and extinction. Many river management projects use stream classification and habitat assessment approaches to design practical solutions to reverse or mitigate adverse effects of flow regime alteration on stream systems. The objective of this study was to develop a methodology to provide a primary identification of physical habitats in an 80-km long segment of the Canadian River in central Oklahoma. The methodology relied on basic geomorphic variables describing the stream and its floodplain that were derived from aerial imagery and Lidar data using Geographic Information Systems. Geostatistical tests were implemented to delineate habitat units. This approach based on high resolution data and did not require in-site inspection provided a relatively refined habitat delineation, consistent with visual observations. Future efforts will focus on validation via field surveys and coupling with hydro-sedimentary modeling to provide a tool for environmental flow decisions.
\end{abstract}

\section{Introduction}

Human activities significantly affect a number of rivers worldwide [1,2], and large alterations of stream systems are causing dramatic species' losses and extinctions [3,4]. Managers attempt to mitigate these effects via strategies aimed to improve the physicochemical habitat, including environmental flows and restoration projects. Physicochemical conditions, a combination of physical and chemical components, define habitats, or the living space of aquatic biota, that are often classified for research and management purposes. Habitat classifications are useful for studying ecological phenomena in rivers [5,6]. Understanding the most important habitats used by fishes and their spatial positioning can provide insight to proper flow management [7]. Habitats are generally described as a hierarchical structure in which each level reveals particular patterns about ecological phenomena. For example Smallmouth Bass use pools more than other habitats at the macro-habitat scale (i.e., multiple patches that comprise a stream reach, often with different water surface slopes and velocity

\footnotetext{
*Corresponding author: 1sguerta@ncsu.edu
} 
patterns [8]). However, at the reach scale, Smallmouth Bass are more abundant in reaches with lower proportions of pool habitat, revealing the importance of habitats that are not directly used by the species [9]. Reaches of varying lengths depending on the study objective, are often described as lengths of stream found between major geomorphic breaks such as changes in slope, valley width and large tributary confluences. The reach scale is the most common scale of observation linking physical and biological processes [10]. Typically, the ecological suitability of any reach to support organisms is dictated by coarser factors such as climate and geology [11]. However, geomorphic and habitat features within these reaches then define the overall distribution and abundances of organisms within the specified study reach. Further, larger reaches $(1-100 \mathrm{~km})$, referenced in ecology as stream segments, are beneficial scales of examination when concerns emerge related to connectivity of habitat [12] For example, some emblematic minnows of the North American Great Plains, USA rely on complexity of habitats across longer stream segments to create suitable drift conditions for their eggs and larvae during development [13].

There are two primary types of habitat assessment protocols that vary in their application depending on the scale of investigation and level of detail needed. Visual-based watershed-scale stream assessment protocols typically in the form of habitat quality indices, are rapid and easy to implement, but rarely provide enough detail to be used for the project design [14]. Transect-based or measurement-based assessments require on-site inspection and are therefore time consuming and labor intensive. Many authors have successfully used physical characteristics of stream to identify habitats $[15,16]$, and the implementation of these approaches could be facilitated as well as their effectiveness improved by using high resolution imagery and Lidar data $[17,18]$ that are nowadays easily available. The objective of this study was to develop a methodology aimed at providing a primary identification of physical habitats in a $80-\mathrm{km}$ long river segment from its physical characteristics derived from high resolution Lidar data, using Geographic Information Systems (GIS) and Geostatistics.

\section{Material and Methods}

\subsection{Study site}

The Canadian River is a 1450-km long, wide and relatively shallow stream flowing in the Southern Great Plains ecoregion of the United States, dominated by sand and mud substrates [19]. Impoundments and increased groundwater pumping have altered flow regimes, stream connectivity and physiochemical patterns of the Canadian River. Stream fragmentation, in combination with other stressors such as altered flow regimes, has led to the rapid decline of pelagic-broadcast spawning fish species emblematic of the Great Plains. Geormorphic complexity is of particular importance for pelagophil species that have specific habitat requirements at different stages of development [13]. Juveniles and adults typically require shallow, slow flowing backwater and side channels with turbid waters, preferentially with sand bottoms. Spawning occurs in the main channel, and eggs and larvae travel with current velocity many kilometers downstream for about three days, requiring substantial lengths of free-flowing water before they are capable of directional swimming.

This study focused on an 80-km long braided meandering segment of the South Canadian River in central Oklahoma, located between Mustang and Purcell USGS gages (Figure 1). Geographical data used to describe this segment included aerial imagery from May 2010 and May 2017, and a composite Digital Elevation Model (DEM) generated from a 2-m resolution Lidar dataset taken in December 2011, with blank areas filled in with a 10$\mathrm{m}$ resolution National Elevation Dataset from 2013. 


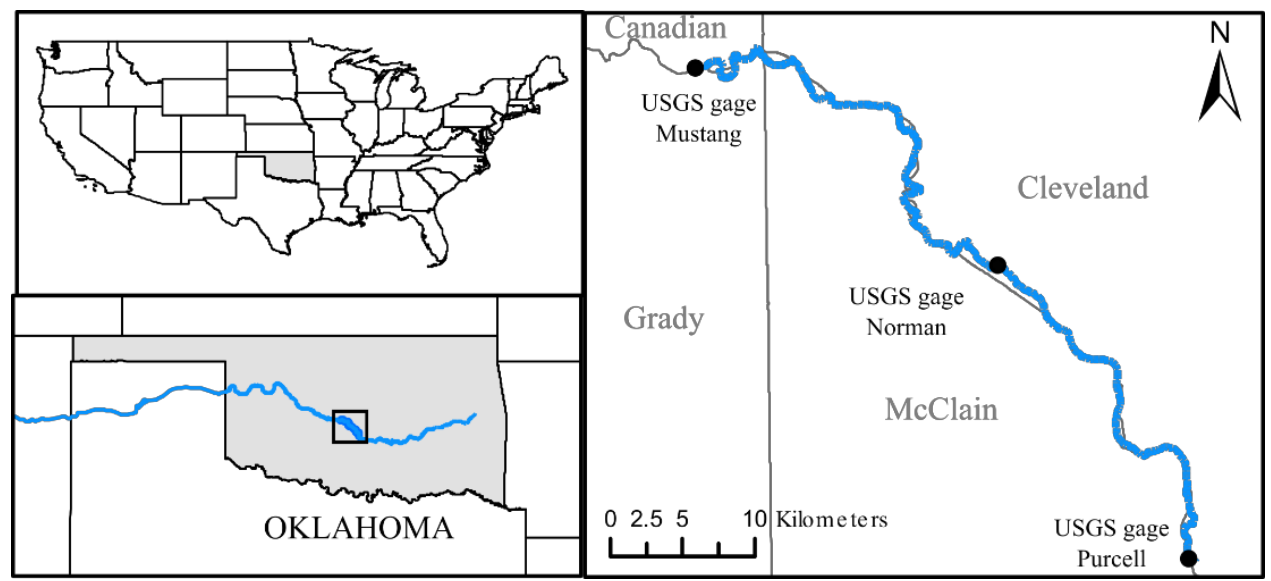

Fig. 1. Location of the studied segment of the Canadian river and USGS gages

\subsection{Stream reach delineation}

Reach boundaries were associated with geomorphic breaks in the floodplain and along the river course. Three variables describing the floodplain and river channel were computed approximately every 300-m using ArcGIS (V. 10.4). Valley width was derived from the composite DEM. The displacement of the centerline between 2010 and 2017 and the cumulated sinuosity were derived from aerial imagery. The cumulated sinuosity at a given river section was calculated as the ratio between the distance along the stream centerline from the river section to the Mustang station to the length of the shortest path between these locations. For each of these three variables, the spatial autocorrelation in the variable was evaluated using a semi-variogram analysis $[16,20]$. An experimental semi variogram was calculated and a spherical semi-variogram model was fit to the experimental semi-variogram using the gstat package of $\mathrm{R}$ (V. 3.3.3). The range is the model parameter that defines the distance at which the variable is no longer correlated. The minimum range fit for the three variables provided an approximation of the dimension of a reach.

The univariate Pettitt [21] test was used to detect breaks in variables, as described in several studies $[20,22]$. The test was applied iteratively until no significant breakpoint was detected, at a significance level $\alpha=0.05$. When breaks were detected at neighboring cross sections, it was assumed that they represented the same transition and they were merged into a unique break.

\subsection{Macro-habitat assessment}

First, the lateral extent of macro-habitat, corresponding to the annual flood channel was determined. The annual flood channel boundaries were assumed to be marked by a continuous vegetation edge, and were delineated using GIS from 2010 aerial pictures.

Longitudinal macro-habitat boundaries, associated with breaks in the river topology, were then identified using the following procedure. The annual flood channel was discretized into a $100-\mathrm{m}$ regular mesh. The total area of each mesh element was calculated. The top width as seen on aerial imagery was measured. Flow conditions displayed on the aerial imagery were close to the average daily flow rate. Then, for each element of the mesh, the reclassify function from ArcGIS was applied to the 2010 aerial image to create a new raster layer by splitting band 1 of the RGB color system into four color categories, each 
corresponding to a topological feature [23]: vegetation, deep water, shallow water (sand visible) and exposed sand (Figure 2). Low density vegetation was sometimes identified as deep water, so the distinction between these two categories was checked visually. Area and proportion within the annual flood channel for each topology class were calculated for each mesh element.

(a)

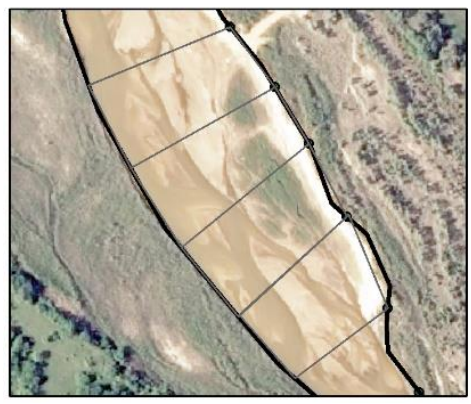

(b)

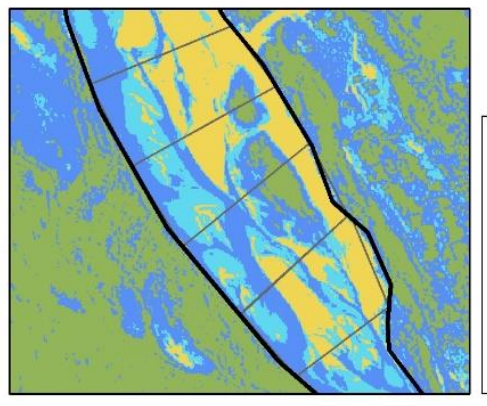

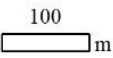

Legend

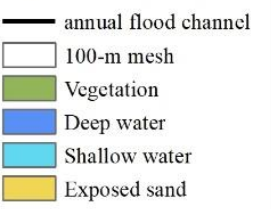

Fig. 2. Conversion of aerial imagery (a) into a raster describing four topology classes (b), annual flood channel delineation and discretization. Note the low-density vegetation that was identified as deep water in the center.

The iterative Pettit test was used to identify breaks in the six variables describing the stream topology (top width, area of the annual flood channel, and proportion of each of the four topographical features). Resulting breaks were added to the reach boundaries previously identified. Similarly, breaks that were detected at neighboring cross sections were merged into a unique break.

\section{Results and discussion}

\subsection{Stream reaches}

In total, thirty-three stream reaches were delineated, with mean and maximum reach lengths of 2,130 $\mathrm{m}$ and $6,480 \mathrm{~m}$, respectively. These dimensions were comparable with the value of the fitted range of the semi variogram model of 5,790 m. Delineated stream reaches were highly variable in length and associated with geomorphic constraints. Reaches boundaries were associated with significant shifts of the main channel, generally observed in higher sinuosity areas, highlighting the meandering pattern of the Canadian river (Figure 3). 
(a)

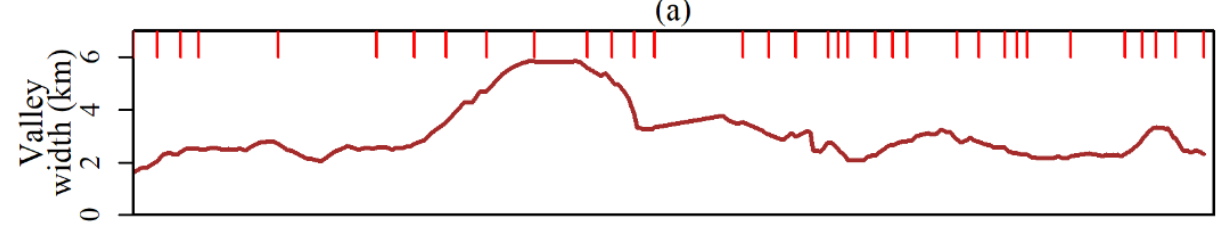

(b)
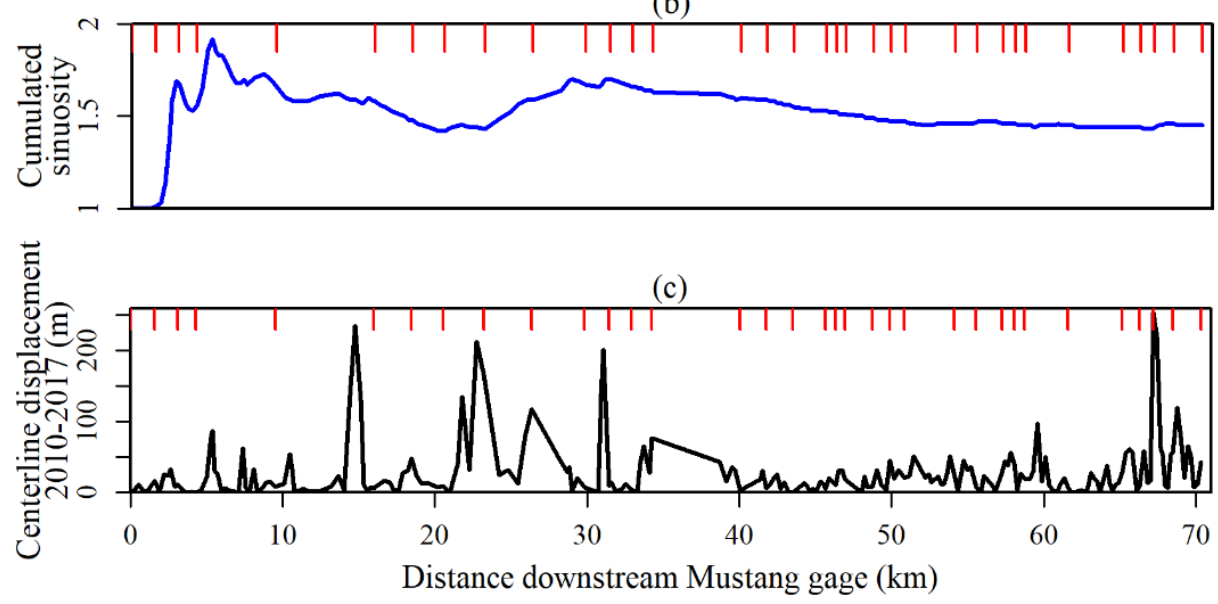

Fig. 3. Longitudinal evolution of geomorphic variables typical of the reach spatial scale and reach boundaries (red upper lines), (a): valley width, (b) cumulated sinuosity, (c) displacement of the centerline between 2010 and 2017.

\subsection{Macro-habitat units}

In total, seventy-nine units were delineated using stream typography and habitat patches repartition variables. Mean and maximum unit length were $750 \mathrm{~m}$ and 3,600 m, respectively. Macro-habitat characteristics were calculated: total area and proportions of each of the four typology class (Figure 4). 


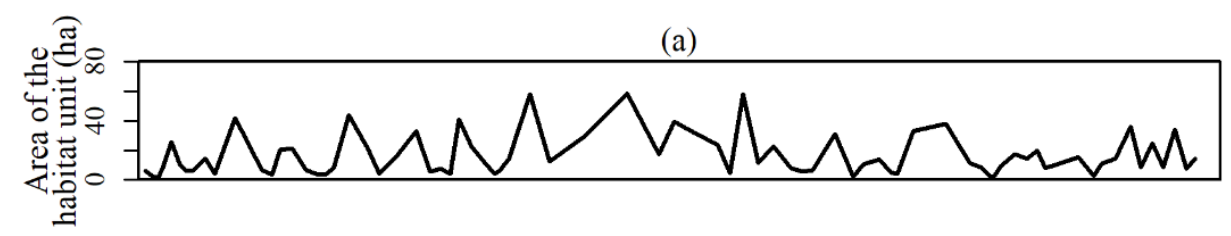

(b)
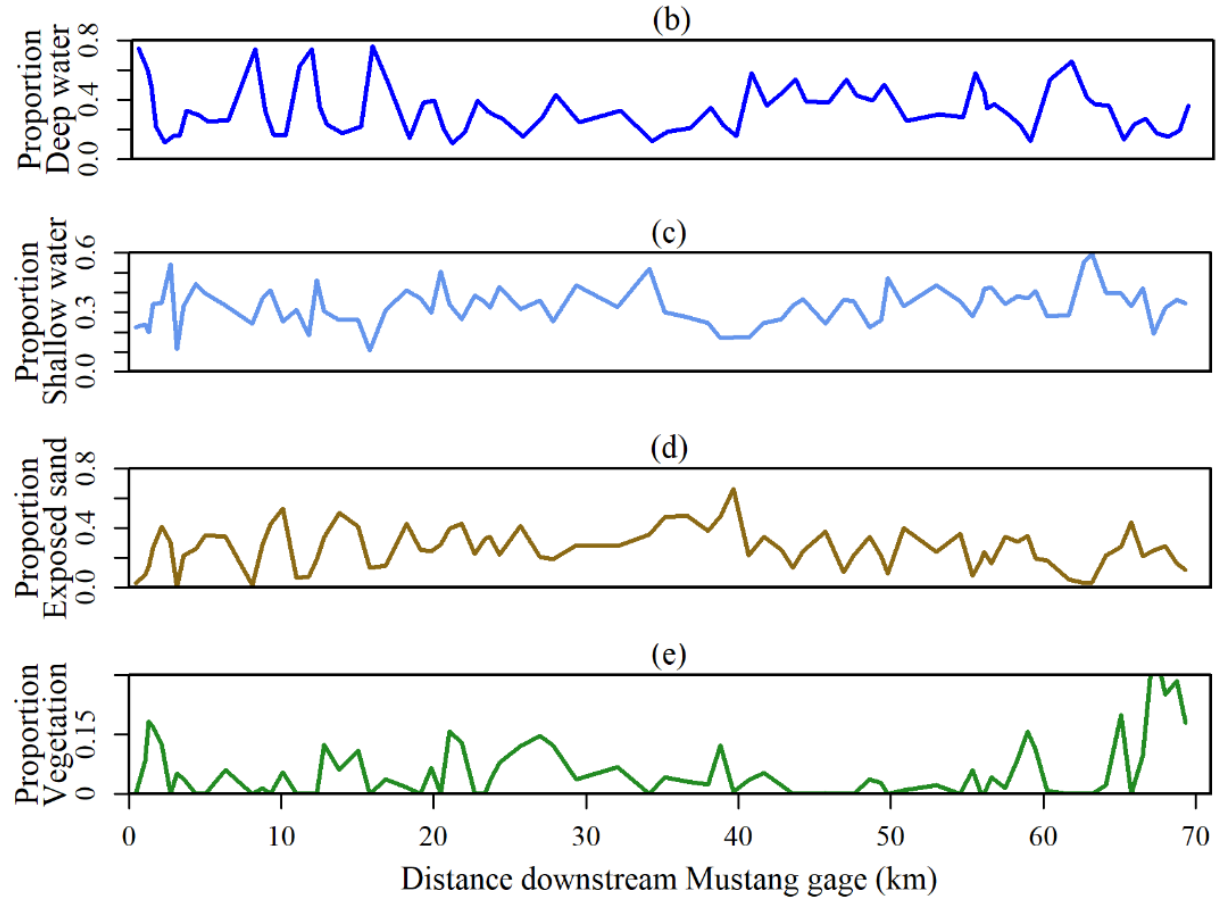

Fig. 4. Macro-habitat units characteristics (each point on the graph corresponds to an unit): (a) total area within the annual flood channel, (b) proportion of deep water, (c) proportion of shallow water, (d), proportion of exposed sand, and (e) proportion of vegetation.

Several types of habitat configuration were identified. Units with low total areas were associated with higher proportions of deep water, and corresponded to narrow stream segments where the average flow channel boundaries were close to the mean annual flood channel (Figure 5 a). Conditions found in these segments are generally problematic for pelogophil species. Higher area units were associated with higher proportions of exposed sand, typically in meandering stream portions (Figure $5 \mathrm{~b}$ ). The geomorphic heterogeneity found in meandering sections can provide suitable habitat conditions for pelagophils. High proportions of shallow water were associated with braiding channel configurations (Figure 5 c). The habitat complexity maintained in the braided channels slows the transport time of the eggs/larvae allowing plenty of time for the eggs to develop in the remaining fragmented reaches. However, a major drought would be problematic if these areas became disconnected via extreme low flows. Riparian vegetation was either located close to the lateral edges of the mean annual flood channel, primarily inside exposed sand areas (Figure $5 \mathrm{~d}$ ), or within multiple channels, on top of stable bars (Figure 5 e). 
(a)

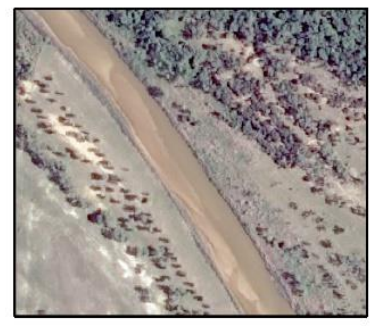

(b)

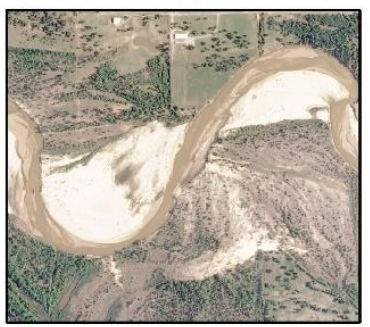

(c)

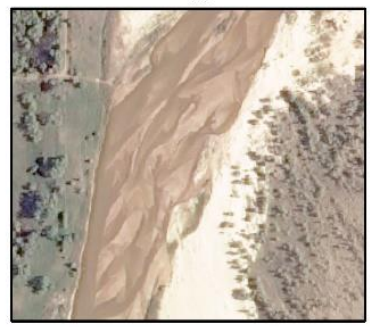

(d)

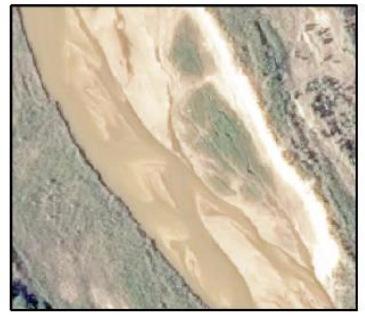

(e)

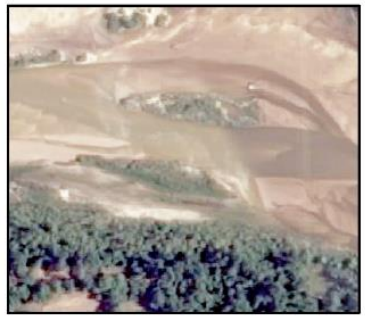

Fig. 5. Typical habitat configurations found in the Canadian River (a) straight and narrow channel, (b) meandering channel, (c) braided channel, (d), lateral vegetation patch, (e) vegetated sand bars.

\section{Conclusion}

A simple methodology was developed to provide a primary identification of physical habitats of an 80-km long segment of the Canadian River in central Oklahoma. The methodology relied on basic geomorphic variables describing the stream and its floodplain; information that can easily be derived from aerial imagery and DEMs. A geostatistical approach was used to delineate habitat units and to identify their main characteristics. Eventually, 33 stream reaches extending between prominent breaks in the stream morphology were identified, and 79 macro-habitat units characterized by specific habitat patches repartition. This approach based on high resolution data provided a relatively refined habitat delineation and did not require in-site inspection. Eventually, the method will be validated against geomorphic field surveys and fish collection records. Habitat identification could also be improved using additional data on stream hydrodynamics (velocities, depths) and water quality (temperature patterns, turbidity).

This delineation provides a functional spatial scale that can be used to assess the impact of human disturbances on stream morphology and habitat suitability at annual to decadal timescales. Especially, it will serve as a reference scale for the development and interpretation of hydro-sedimentary modelling of the study reach, that will be used to provide tools for developing environmental flows to maintain channel complexity and increase habitat suitability for pelagophil species.

This research is a contribution of North Carolina State University and the Oklahoma Cooperative Fish and Wildlife Research Unit (U.S. Geological Survey, Oklahoma Department of Wildlife Conservation, Oklahoma State University, and Wildlife Management Institute cooperating). Funding 
was provided by the North Carolina Agricultural Research Service. Any use of trade, firm, or product names is for descriptive purposes only and does not imply endorsement by the U.S. Government.

\section{References}

1. T.P. Barnett, D.W. Pierce, H.G. Hidalgo, C. Bonfils, B.D. Santer, T. Das, G. Bala, A.W. Wood, T. Nozawa, A.A. Mirin, D.R. Cayan, Science, 319(5866):1080-1083 (2008)

2. S. Wang, B. Fu, S. Piao, Y. Lü, P. Ciais, X. Feng, Y. Wang. Nat. Geosci., 9(1):38-42 (2015)

3. M.P. Marchetti, P.B.Moyle, Ecol. Appl., 11(2): 530-539 (2001)

4. S. J. Wenger, D.J. Isaak, C.H. Luce, H.M. Neville, K.D. Fausch, J.B. Dunham, D.C. Dauwalter, M.K. Young, M. M. Elsner, B.E. Rieman, A.F. Hamlet, J.E. Williams, PNAS, 108(34):14175-14180 (2011)

5. J.R. Thomson, M.P. Taylor, K.A. Fryis, G.J. Brierley Aquatic Conser.: Mar. Freshw. Ecosyst. 11:373-389 (2001)

6. C. Parker, N.J. Clifford, C.R. Thorne, River Res. Applic. 28:1708-1725 (2012)

7. S.K. Brewer, R.A. McManamay, A.D. Miller, R. Mollenhauer, T. Worthington, T. Arsuffi,. Environ. Manage. 58:175-192 (2016)

8. C.A. Frissell, W.J. Liss, C.E. Warren, M.D. Hurley, Environ. Manage. 10(2):199-214 (1986)

9. S.K. Brewer, N. Am. J. Fish. Manag., 33(2):351-358 (2013)

10. D. Tetzlaff, C. Soulsby, P.J. Bacon, A.F. Youngson, C. Gibbins, I.A. Malcolm, Hydrol. Process., 21:1385-1389 (2007)

11. H.B.N. Hynes, Proceedings of the International Association of Theoretical and Applied Limnology 19:1-15 (1975)

12. K.D. Fausch, C.E. Torgersen, C.V. Baxter, H.W. Li. Bioscience 52(6): 483-498 (2002)

13. T.A. Worthington, A.A. Echelle, J.S. Perkin, R. Mollenhauer, N. Farless, J.J. Dyer, D. Logue, S.K. Brewer, Fish and Fisheries, 00:1-37 (2017)

14. D.E. Somerville, B.A. Pruitt, Physical Stream Assessment: A Review of Selected Protocols for Use in the Clean Water Act Section 404 Program. EPA 843-S-12-002 (2004)

15. G.J. Brierley, K.A. Fryis, Environ. Manage. 25(6):661-679 (2000)

16. J.S. Clark, D.M. Rizzo, M.C. Watzin, W.C. Hession, River. Res. Applic. 24:885-899 (2008)

17. Z.H. Bowen, R.G. Waltermire, J. Am. Water Resour. Assoc. 38(1):33-41 (2002).

18. D.M. Heeren, A.R. Mittelstet, G.A. Fox, D.E. Storm DE, A.T. Al-Madhhachi, T.L. Midgley, A.F. Stringer AF, K.B. Stunkel KB, R.B. Tejral, Trans. ASABE 55(3): 957968 (2012).

19. A.K. Tyagi, P. Poudyal Channel-bed degradation in major Oklahoma Streams, Volume IV of V: Canadian River, (Oklahoma State University, 2007)

20. A. Latapie, B. Camenen, S. Rodrigues, A. Paquier, J.P. Bouchard, F. Moatar, Catena, 121:1-12 (2014)

21. A.N. Pettitt, J. R. Stat. Soc. Ser. C Appl. Stat. 28(2):126-135 (1979)

22. V. Martínez-Fernández, J. Solana-Gutiérrez, M. González del Tánago, D. García de Jalón, Geomorphology, 253:38-47 (2016)

23. T.A. Worthington, S.K. Brewer, N. Farless, T.B. Grabowski, M.S. Gregory, Plos One, 9(5):1-9 (2014) 